\title{
Effects of Lesions of Temporal-Parietal Junction on Perceptual and Attentional Processing in Humans
}

\author{
Lynn C. Robertson, ${ }^{1}$ Marvin R. Lamb, ${ }^{1}$ and Robert T. Knight ${ }^{2}$ \\ Department of 'Psychiatry and 'Neurology, University of California, Davis, Veterans Administration Medical Center, \\ Martinez, California 94553
}

\begin{abstract}
When stimuli with larger forms (global) containing smaller forms (local) are presented to subjects with large lesions in the right hemisphere, they are more likely to miss the global form than the local form, whereas subjects with large lesions in the left are more likely to miss the local than the global form. The present study tested whether the global/local impairment in subjects with posterior lesions was due to deficits in controlled attentional processes, passive perceptual processes, or both. Attentional control was examined by measuring reaction time changes when the probability of a target appearing at either the global or local level was varied. Patients with unilateral right or left lesions centered in temporal-parietal regions and age-matched controls served as subjects. Because neurophysiological and neuropsychological evidence have implicated temporal regions in visual discrimination and inferior parietal regions in the allocation of attention to locations in the visual field, patients with left hemisphere lesions were further subdivided into those with lesions centered in the superior temporal gyrus (LSTG) or rostral inferior parietal lobule (LIPL). Patients with right hemisphere injury could not be analogously subdivided. The results revealed that the LSTG group was able to control the allocation of attention to global and local levels normally, while the LIPL group was not. In contrast, the LSTG group showed a strong baseline reaction time advantage toward global targets, while normals and the LIPL group showed no advantage toward one level or the other. Finally, the perceptual component was affected differentially by lesions in the right hemisphere and LSTG, with lesions in the left favoring global targets and lesions in the right favoring local targets. These findings indicate that the hemispheric global/ local asymmetry is due to a perceptual mechanism with a critical anatomical locus centered in the STG.
\end{abstract}

Physiological and behavioral data have implicated the posterior parietal regions in visuospatial analysis. Ablation studies in primates have shown that inferior parietal damage disrupts the ability to determine the spatial relations between objects in the visual field but not their identity (Pohl, 1973; Ungerleider and

\footnotetext{
Received Aug. 6, 1987; revised Nov. 23, 1987; accepted Jan. 13, 1988.

The present work was supported by the Research Service of the Veterans Administration Medical Center and by NIAAA Grant AA06486 to L.C.R. and by NINCDS Grant NS21135 to R.T.K. We wish to thank Clay Clayworth and Robert Frey for development of computer reconstruction software.

Correspondence should be addressed to Lynn C. Robertson, Research Service 151, Veterans Administration Medical Center, 150 Muir Road, Martinez, CA 94553 .

Copyright (C) 1988 Society for Neuroscience $0270-6474 / 88 / 103757-13 \$ 02.00 / 0$
}

Mishkin, 1982). In addition, single-cell recordings in monkey have shown that cells in the same area respond to task-relevant visual targets. These effects occur independently of eye movements and have been attributed to selective attention (Lynch et al., 1977; Lynch 1980; Goldberg, 1982). Conversely, single-cell recordings from caudal temporal regions have revealed lightsensitive cells in the superior temporal sulcus that do not appear to be modulated by the motivational state of the animal (Zeki, 1974; Desimone and Ungerleider, 1986; Ungerleider and Desimone, 1986), and lesions in this area in primates result in deficits in object discrimination but not spatial relations (Ungerleider and Mishkin, 1982). Behavioral data in humans are consistent with these findings. Lesions in parietal areas impair responses to relevant stimuli appearing in unexpected locations in the visual field (Posner et al., 1984), and performance on spatial-relations tasks can be altered with parietal damage, while the ability to identify objects can be altered with temporal damage (Newcombe and Russell, 1969).

Although primate studies have not focused on left/right differences in parietal regions, functional deficits in attention and spatial analysis in humans are typically hemisphere specific. It is well known, for instance, that right parietal damage in humans is more likely than left to result in hemispatial neglect (see Mesulam, 1981, for a review) and that deficits in visuospatial tasks are differentially affected by unilateral damage to one hemisphere or the other. For instance, data from our laboratory have shown that large lesions in the distribution of the right middle cerebral artery disrupt the organization of a global form like that shown in Figure 1 (the S). Conversely, large lesions in the left hemisphere are more likely to affect the ability to respond to local forms like the Es in Figure 1 (Robertson and Delis, 1986; Delis et al., 1986). These effects occur both when the

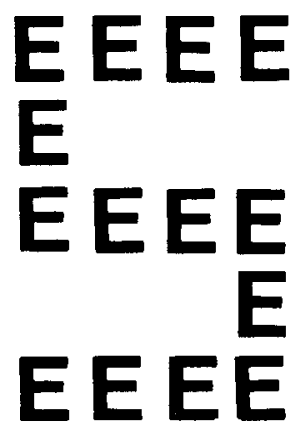

Figure 1. Example of a large form containing smaller forms. The S represents the global level and the E's the local level. 
patterns are letters and when they are geometric forms. It is not known to what extent attentional and/or perceptual functions are involved in these differences. One of the goals of the present study was to address this question with patients who have lesions centered in caudal temporal or inferior parietal regions.

A great deal is known about processing global and local levels in normal populations from studies in cognitive psychology, and it has become evident that both passive perceptual processes and controlled attentional processes are involved (Navon, 1977; Kinchla and Wolfe, 1979; Martin 1979; Hoffman, 1980; Palmer, 1982; Sergent, 1982; Kinchla et al., 1983; Pomerantz, 1983; Robertson and Palmer, 1983; Lamb and Robertson, 1988). Early studies demonstrated that normals could identify the global form before the local form. Subsequent studies have shown that this global advantage can be changed to a local advantage by parametric variations that are known to affect perception (e.g., visual angle, number of local elements, retinal locus, symmetry, shape). Others have shown that it can be changed by manipulations known to affect the distribution of attention. One such study is especially relevant for the present purposes. Kinchla et al. (1983) presented global/local patterns to normal subjects but varied the probability that a target would appear at a given level. When a target was more likely to occur at one level (either global or local), detecting a target at that level was better than detecting a target at the less probable level. There was a trade-off in detecting global and local targets despite the fact that stimulus parameters were held constant, suggesting that the allocation of attention to the 2 levels differed across conditions to optimize performance. We have replicated and extended these findings using a reaction time paradigm (Lamb and Robertson, 1987) and have found that both perceptual and attentional processes must be taken into consideration (Lamb and Robertson, 1988). Our data also suggested that the type of attention involved was one that controls the allocation of attention over the visual field by varying the visual area monitored. One question addressed in the present study is whether controlled attentional mechanisms associated with parietal regions and visual discrimination mcchanisms associated with caudal temporal regions can produce the global/local dissociation observed in patients with left or right hemisphere lesions. A second question is whether a global/local dissociation will occur when the more sensitive measure of reaction time is used, when subjects with focal lesions are carefully selected, and when the subjects are well beyond the acute stage.

Before we discuss the methods, it is important to understand what type of attention we are studying in the present experiment. The concept of attention has been used in various ways (see Parasuraman and Davies, 1984, for an overview), but the type of attention under consideration in the present study is the controlled distribution of attention over the visual field. Controlled processes are by definition under the subject's control and can be contrasted to mechanisms associated with an orienting response or an overlearned automatic response. The most distinguishing characteristic of controlled processes is that whenever attention is divided between attributes of a stimulus, the more attention given to one part of the stimulus, the less will be available for others. The result will be a slower response to an attribute as less attention is allocated to that attribute and a faster response to an attribute that receives more attention. This trade-off is, in fact, one of the main defining properties of controlled attentional processes and has been extensivcly uscd as a diagnostic of controlled attention (see Shiffrin and Schnei- der, 1977; Schneider and Shiffrin, 1977; Kahneman and Treisman, 1982; Posner and Rafal, 1987).

Attentional mechanisms, of course, must interact with perceptual mechanisms. It does not matter how widely attention is distributed if the perceptual channel itself is impaired. It is also the case that perception requires the coordination of several mechanisms, some of which could directly account for the global/local dissociation as seen with left/right lesions. In sum, the effects of unilateral hemisphere lesions on response to patterns with global and local information could be due to alterations in direct automatic encoding of stimulus features (what we are here calling a perceptual mechanism), to controlled attentional mechanisms that govern the extent of space to be monitored, or to both. It is critical that the nature of the behavioral deficit be specified precisely if the functional aspects of the neural systems involved are to be understood.

In the present study, subjects with left or right temporalparietal damage and controls were presented stimuli with global and local levels and were asked to detect a target appearing at one level or the other. Subjects with left hemisphere damage were further subdivided into those with lesions centered in the supcrior tcmporal gyrus (LSTG) and those with lesions centered in inferior parietal lobule (LIPL) on the basis of lesion location as determined by CT scan as well as electrophysiological data from separate studies (Knight et al., 1987a, b). Subjects with right temporal-parietal lesions (RTP) were more heterogeneous and could not be separated on these bases. There were 3 attention conditions, all of which required dividing attention between the 2 levels. In the No-bias condition, the target appeared equally often at each level. In the Global-bias condition, the target appeared at the global level $75 \%$ of the time, and in the Localbias condition, the target appeared at the local level $75 \%$ of the time. For subjects who can effectively distribute attention between the 2 levels, performance should vary systematically in the different biasing conditions. Conversely, the biasing conditions should have little or no effect on subjects with an attentional deficit.

\section{Materials and Methods}

Subjects. The patients were all selected on the basis of evidence of a single focal lesion in the temporal-parietal region. There were 5 LIPL subjects, 5 LSTG subjects, 6 RTP subjects, and 9 normal control subjects. The clinical specifications for each subject as well as the location and extent of lesions, visual acuity, and field deficits are presented in Table 1. All subjects were male except for a female RTP patient (KE). The average age of the control subjects was $52.4(\mathrm{SD}=12.4)$, and the average age of the patients was $54.5(\mathrm{SD}=12.0)$. Twelve subjects had suffered stroke, 2 (KE and JC) had tumors surgically resected but had been stable for over 5 years with no evidence of tumor regrowth, one (MK) had received a shrapnel wound 10 years earlier, and one (GB) had surgical resection of hemorrhagic temporal-parietal cortex subsequent to a posttraumatic subdural hematoma 13 years earlier. Subjects were screened for drug and alcohol addiction, dementia, psychiatric disorder, marked aphasia, and major medical illness independent of their neurological problems. Patients with evidence of increased intracranial pressure or shift of midline structures were excluded. All subjects had normal or corrected to near-normal visual acuity as measured by Snellen chart. One of the RTP subjects had some evidence of mild neglect as measured by Albert's line crossing test. Details of the occurrence of neglect during the onset of neurological problems were unknown. Visual fields were tested by confrontation, and although several subjects did have field defects, they were equally distributed between the left and right hemisphere damaged groups. Because the patients were also being run in auditory experiments in a different laboratory, they were screened for hearing loss. One patient (RH) had hemiparesis due to subcortical extension into the centrum semiovale, but the rest 
had no evidence of hemiparesis. All subjects were at least 1 year post insult in order to optimize baseline stability. Five of the LHD patients (DL, LG, BB, RW, FO) showed mild to moderate signs of aphasia upon neurological examination. One had suffered a transient episode of aphasia postinsult but no longer exhibited any evidence of aphasia (FA); the rest had never shown evidence of aphasia.

The 9 normal control subjects were sex- and age-matched to the patient groups. They all had normal or corrected-to-normal vision. Both controls and patients were paid for their participation.

Lesion location. Lesion location was verified by CT scan in all patients. Location was judged by 3 independent raters, 2 of whom were naive to the hypotheses of the present study, and agreed-upon areas were reconstructed by a neurologist (R.T.K.) onto axial templates drawn from an atlas (DeArmond et al., 1976). Individual and group lateral reconstructions were then computed from the axial sections (Frey et al., 1987). Figure 2 shows the average reconstruction for patient groups divided into lesions centered in the left superior temporal gyrus and adjacent caudal inferior parietal lobe (LSTG), those centered in the left rostral inferior parietal lobule (LIPL), as well as for those with lesions in right temporal parietal region (RTP). Because of fewer subjects and greater homogeneity, the RTP group could not be separated into subgroups on the same basis as the patients with left hemisphere lesions. As can be seen in the individual reconstructions shown in Table 1, one LIPL patient had minimal lesion extension into the posterior superior temporal plane (FO), and several LSTG patients had lesions extending into caudal LIPL. However, the LIPL and LSTG patients were also differentiated on the basis of electrophysiological data. All LSTG patients had reduced or absent auditory evoked potentials generated by superior temporal plane structures (Knight et al., 1987a), as well as reductions of the cognitive P300 potential (Knight et al., 1987b). Conversely, LIPL patients had normal $\mathrm{P} 300$ potentials and temporal plane auditory evoked potentials but reduced N200 responses.

Stimuli. A set of hierarchically formed letters with global and local levels was constructed. Global letters were composed of local letters arranged appropriately and placed in a 4 (horizontal) $\times 5$ (vertical) matrix. The local letters subtended approximately $1.2^{\circ}$ visual angle vertically and $0.8^{\circ}$ horizontally. The global letters subtended about $6.6^{\circ}$ visual angle vertically and $4.3^{\circ}$ horizontally. The stimuli were generated on a Princeton Graphics SR-12 monitor controlled by an IBM PC-XT computer with color Sigma Designs Graphics Dazzler I and Enhancer cards and appeared as white on a dark background. Stimulus timing (onset, offset, and duration) was tied to the vertical sync pulse. All other events (responses, ITI, etc.) were timed using the 8253 chip set to a 1 msec time base. The status of the response key was monitored via the game port.

The letters $\mathrm{S}$ and $\mathrm{H}$ served as targets, while $\mathrm{A}$ and $\mathrm{E}$ served as nontargets. These letters appeared in all possible combinations equally often with the exception that each stimulus contained either an $\mathrm{S}$ or an $\mathrm{H}$ but not both.

Procedure. Subjects sat with the back of their head against the back of a large easy chair. On each trial a tone sounded for $500 \mathrm{msec}$. This was followed, $100 \mathrm{msec}$ after tone offset, by one of the hierarchical stimuli. The stimulus figure was presented for $100 \mathrm{msec}$, and it was located in the center of the screen. A new trial began $1000 \mathrm{msec}$ after the subjects' response. Each block began with 4 warm-up trials that were not included in the analysis. In one block (No-bias condition), the target (S or H) appeared equally often at the global and local levels. In another block (Global-bias condition), the target occurred at the global level $75 \%$ of the time and at the local level $25 \%$ of the time. In yet another block (Local-bias condition), the target occurred at the local level $75 \%$ of the time and at the global level $25 \%$. In all cases the subject was instructed to respond by pressing one key on a response box if an $S$ was present and another if an $\mathrm{H}$ was present whether at the global or local level. Each letter was mounted adjacent to the appropriate key. Subjects used the hand ipsilateral to the lesion and responded with their index and middle fingers. Six of the control subjects responded with their left hand and 3 with their right hand, a ratio determined to be nearly proportional to the different hands the patients were required to use.

Stimuli were randomly presented with the following constraints. In the No-bias condition there could be no more than 4 consecutive same letter targets, no more than 4 consecutive same letter nontargets, and no more than 4 consecutive targets at a given level. In the Global-bias and Local-bias conditions, the only restriction was that a target appear at the biased level for the first 10 trials in an attempt to draw attention to that level. In order to avoid differences due to comprehension, the probabilities were not mentioned to the subjects, nor were they told that a change in probabilities would occur. Reaction times and errors were recorded.

Experimental trials were preceded by a comprehension task and practice trials. In the comprehension task, patterns from the stimulus set were presented on the screen one at a time for as long as it took the subject to respond correctly. Subjects stepped through at least 24 such trials but continued until it was clear they understood the response rule. The subject then experienced 48 practice trials in which the experimental parameters were used. Practice was given to reduce variability in response time and to allow subjects to become used to the procedure.

Subjects participated in 408 experimental trials with a break of at least $5 \mathrm{~min}$ in the middle. The duration varied depending on the readiness of the subject to continue. No break lasted longer than $20 \mathrm{~min}$. Each subject was run first in the sequence of either Local-bias/No-bias/ Global-bias or Global-bias/No-bias/Local-bias to control for the effects of practice on global and local responding. Whatever sequence a subject received before the break, they received the opposite sequence after. The practice block was given before each sequence.

Subjects were told that both reaction times and errors were important, so that they should respond as rapidly as possible while making few errors. In all conditions they were told to respond to the targets regardless of the level at which they occurred.

\section{Results}

Median response times for correct responses were calculated for each subject with an average of 30 observations per cell per subject. The data were then analyzed using analysis of variance tcchniques. The mean of the median response times collapsed over subjects are presented in Figure 3 for all 4 groups. The $\mathrm{RTP}_{2}$ data to the right will be discussed later. The individual response times for each patient are presented in Table 2 collapsed over target letter.

In the overall analysis a mixed design ANOVA was used with groups as the between-subjects factor (Control, LIPL, LSTG, and RTP) and target level (Global vs Local), target letter (H or $\mathrm{S}$ ), and bias condition (No-bias, Global-bias, or Local-bias) as within subject factors. We report the effects of the overall analysis in condensed form first and elaborate on them in subsequent sections.

The only significant main effect observed was for Group, $F$ $(3,21)=15.10, p<0.001$. Longer reaction times were found in the LSTG group and shorter reaction times in the LIPL group compared with controls. The control and RTP groups did not differ from one another in this respect.

In addition to the main effect of group, there were 3 significant interactions in the overall analysis. The first was a Target level $\times$ Group interaction, $F(3,21)=3.96, p<0.02$, indicating that the groups differed in the degree to which reaction time favored the global or local level. The second was a Target level $\times$ Bias interaction, $F(2,42)=58.90, p<0.001$, revealing that the favored level changed as the target probabilities changed. The third was a Target level $\times$ Bias condition $\times$ Group interaction, $F(6,42)=3.73, p<0.01$, meaning that the Target level $\times$ Bias interaction differed across groups. No other significant effects were found in the overall analysis. These effects will be discussed in turn as they relate to attention, perception, and lesion location.

\section{Target level $\times$ Bias interactions and attentional control}

As can be seen in Figure 3, the biasing conditions were effective in producing performance trade-offs as the target probabilities changed. This is best observed in the control group, in which the crossover is practically symmetrical. Relative to NB, reaction times were faster to local targets in LB and to global targets in GB. Equally important as an indicator of attentional control are that reaction times decreased to global targets in LB and to 
Table 1. Clinical data for each patient

Left Temporal Parietal

Sex/Age/

RG $62 / M$

IIPL

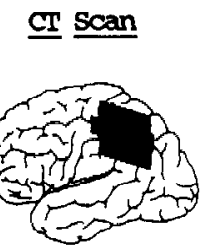

ES $69 / \mathrm{N}$

ITIL

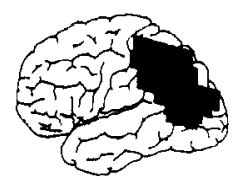

Fo $57 / \mathrm{M}$

LIPL

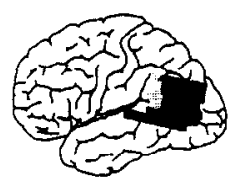

FA $60 / \mathrm{M} /$

LIPL

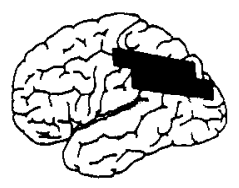

MR $40 / \mathrm{M}$

ITPL

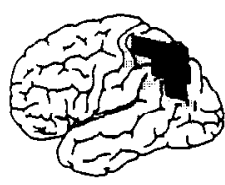

Mild I/R difficulty

Mild finger agnosia, acalculia

Clinical Deficits

Normal

CVA/ 1 Y

CVA/8 YNS

Conduction aphasia, stuttering

SFormal

CVA/5 YRE

Trauma/19 yrs

$20 / 20-20 / 20$

Full $\frac{\text { Visual }}{O D} \frac{\text { Aculty }}{\underline{O S}} \quad \frac{\text { Visual Field }}{\underline{O D}} \frac{\underline{O S}}{}$

$20 / 20-20 / 25$

Full
CVA/4 yrs 20/20 - 20/25 Full

20/20 - 20/20 Full
Fw $53 / M$ ISTG

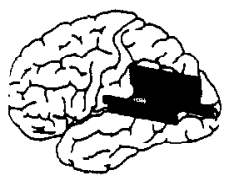

DL $52 / \mathrm{NL}$ LSTG

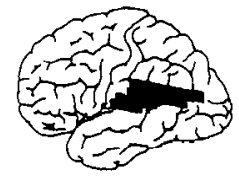

concuction aphasia

Stuttering, Gerstmann's sundrame, ISTG

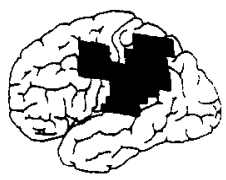

CVAV Y Ys

Nild recept
stuttering

$20 / 20-20 / 40$<smiles>Cc1ccc(I)c([C@@H]2C[C@H](C)CC[C@H]2C)c1</smiles>

CVAl yr

$20 / 20-20 / 20$

Full

CVA 5 yrs

$20 / 30-20 / 20$

Full

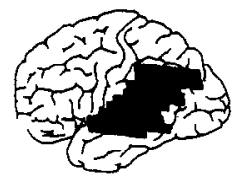


RH $60 / 24$

ISTG

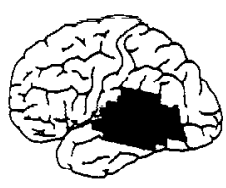

HS

$72 / \mathrm{M}$

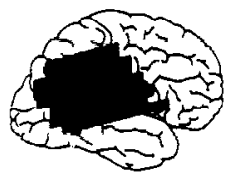

FM

$66 / 14$

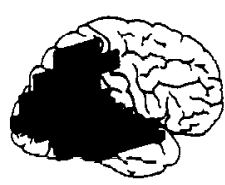

KE

$36 / F /$
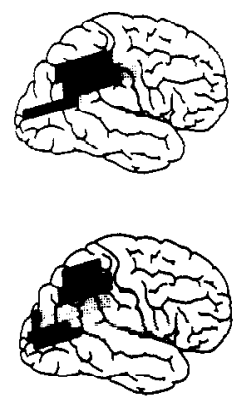

GB

$38 / \mathrm{W}$
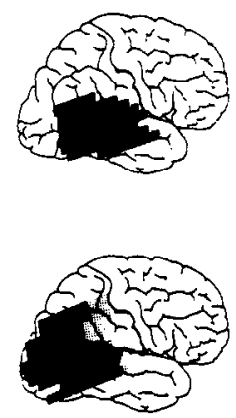

Normal

Decreased proprioception L side, mild pseudo atheotosis I side

Normal

Anomia, anterograde memory difficulty

CVA/21 Yrs

Right Teuporal Parietal

Mild agraphesthesia, astereognosis I side

Aprosodic, I henianesthesia, agraphesthesia, astereognosis, pseudoathetosis

Astereognosis I hand
CVA12 Yrs

$20 / 20-20 / 20$

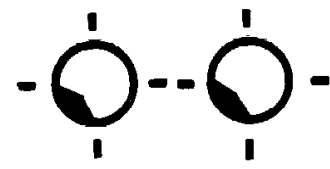

CVA 7 yrs

Tumor resected 12 yrs

CVA/ 1 yr

$20 / 40-20 / 30$

Full

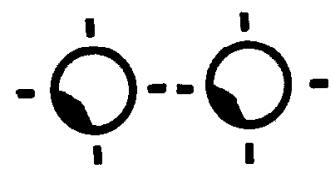

$20 / 20-20 / 20$

Trauma/13 yrs
$20 / 20-20 / 20$

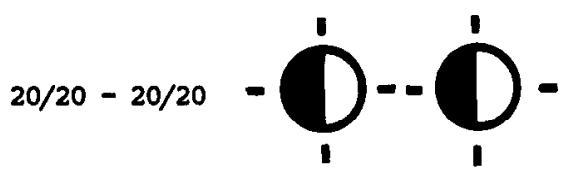

Tumor
10 yes local targets in GB. Planned single degree of freedom comparisons (Keppel, 1973) confirmed all these differences statistically (all $p<0.02$ ). These data strongly support the role of attentional control in processing global and local levels of a stimulus and demonstrate that normal subjects were able to direct more attention to the level at which the target was most likely to appear.

Because the Target level $\times$ Bias condition interaction differed across groups, we compared each of the patient groups with the control group to determine which groups deviated from the normal pattern. A difference between a patient and control group would indicate a dysfunction in the ability to allocate attention normally to global and local levels of the stimulus patterns. These comparisons revealed a significant Target level $\times$ Bias condition $\times$ Group interaction only between LIPL and controls, $F(2,24)=4.71, p<0.02$. As can be seen in Figure 3 , the LB and GB functions for the LIPL group were flattened relative to controls. In fact, when the Target level $\times$ Bias condition interaction indicative of controlled attention was analyzed in the LIPL group alone, it was not significant, whereas it did reach significant levels for all other groups (all $p<0.01$ ).

\section{Target level $\times$ Group interactions and relative global/local advantage}

In addition to the differences observed between groups in the crossover effects, there were also differences in relative reaction 


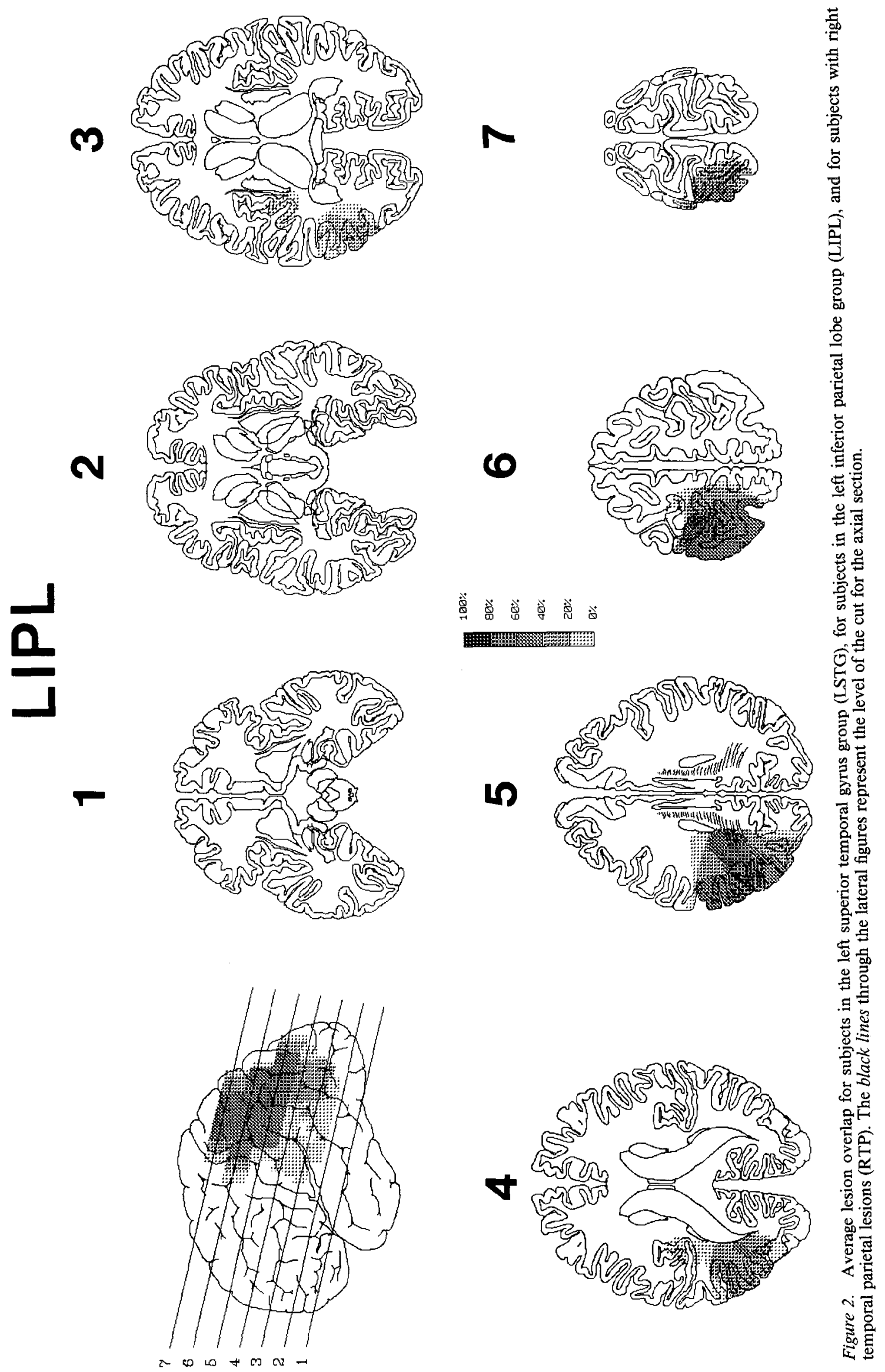



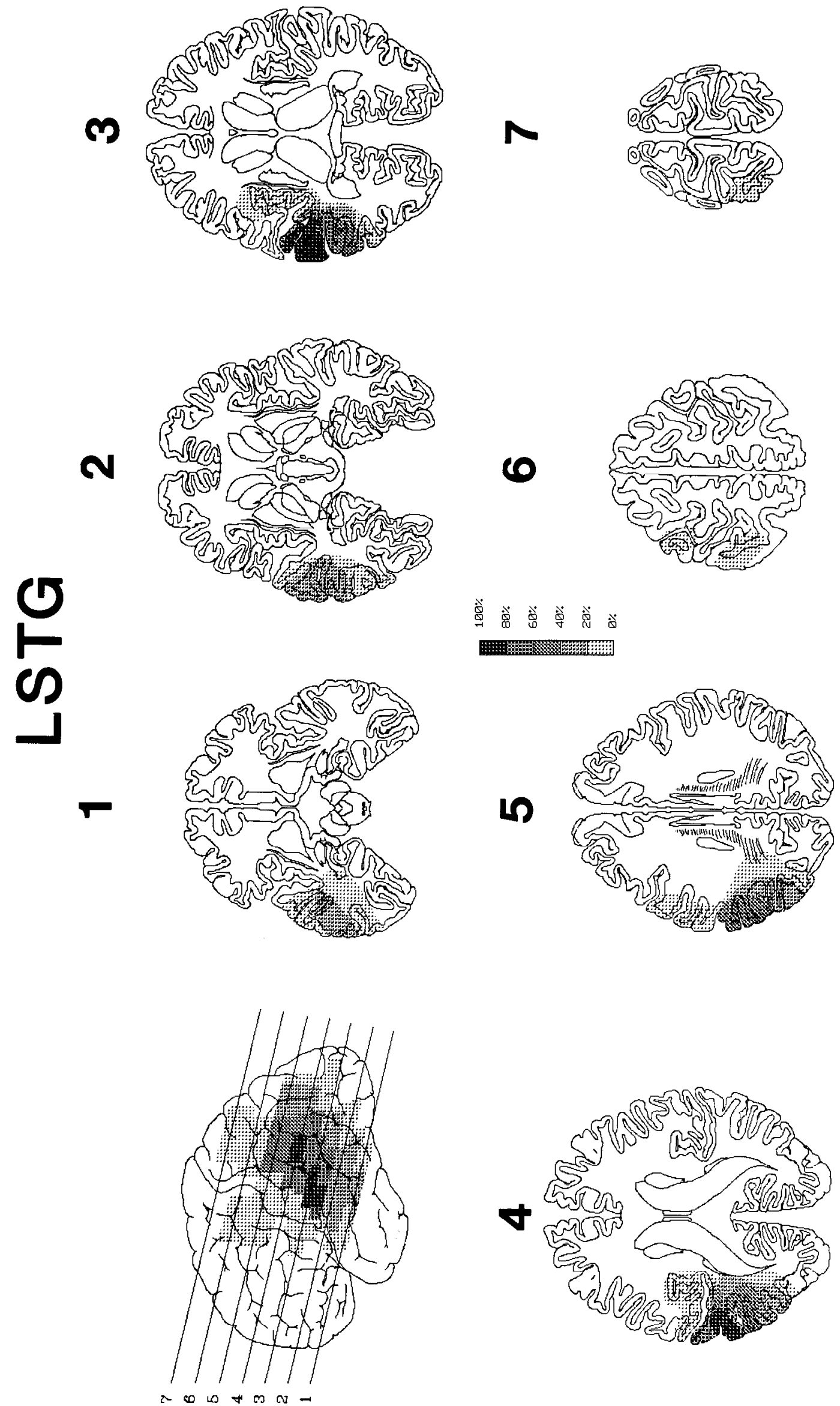

A 
ค)

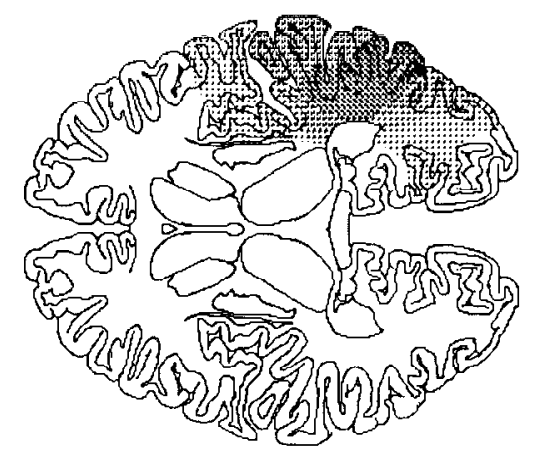

$\mathbf{N}$

\section{a $\stackrel{F}{\square}$}

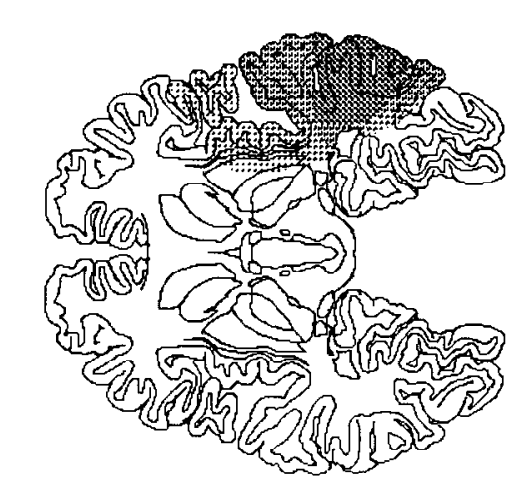

$\infty$

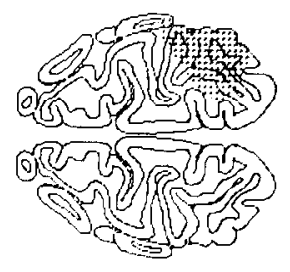

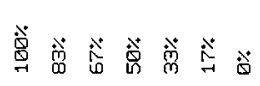

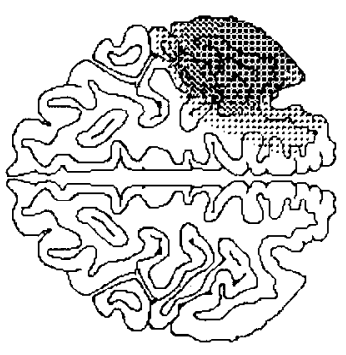

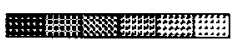

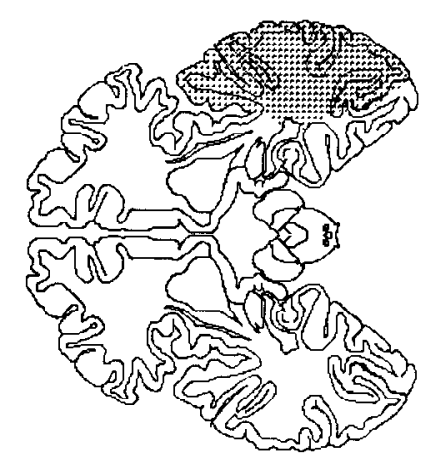

10
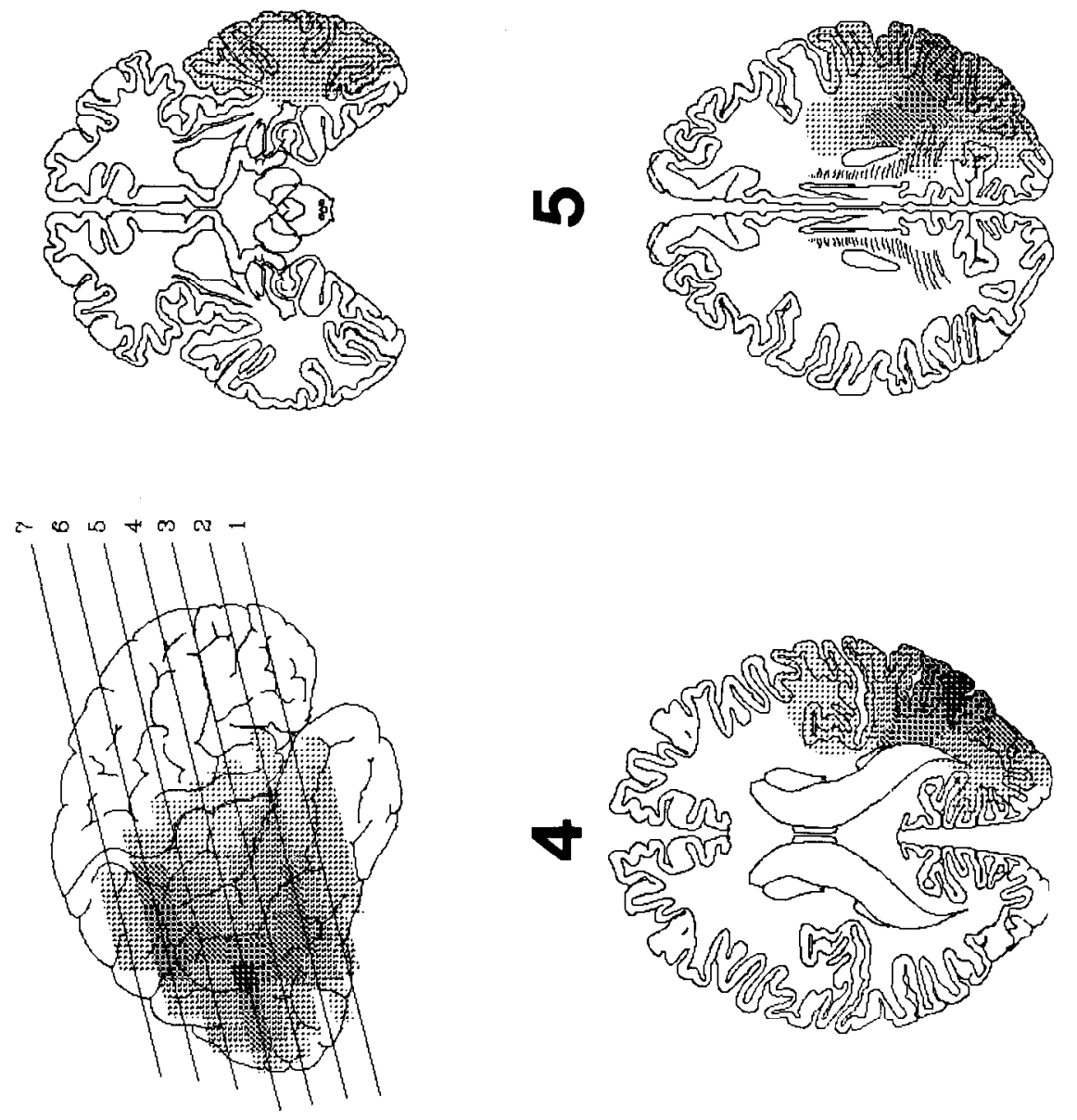

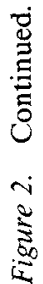


Controls $(\mathrm{N}=9)$

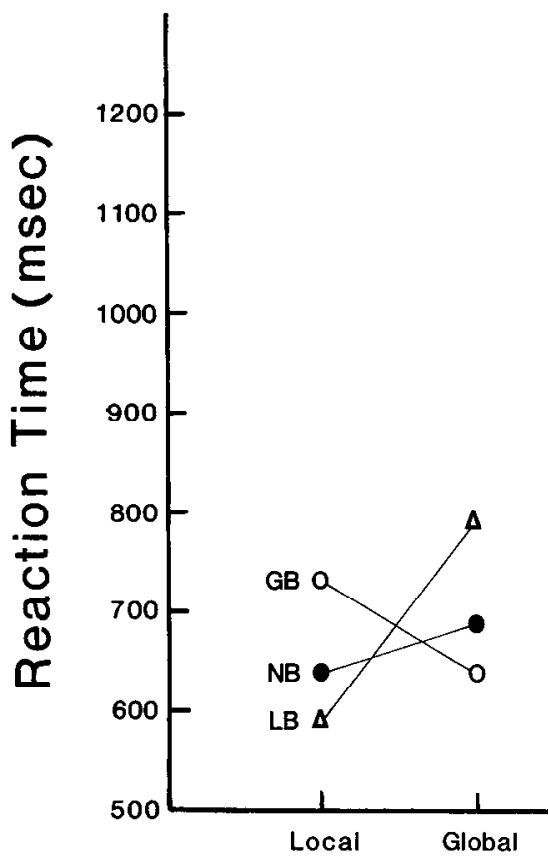

LIPL $(\mathrm{N}=5)$

LSTG $(N=5)$

GB $Q$

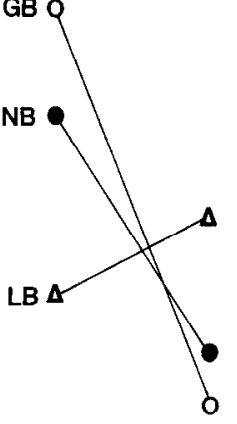

GBO

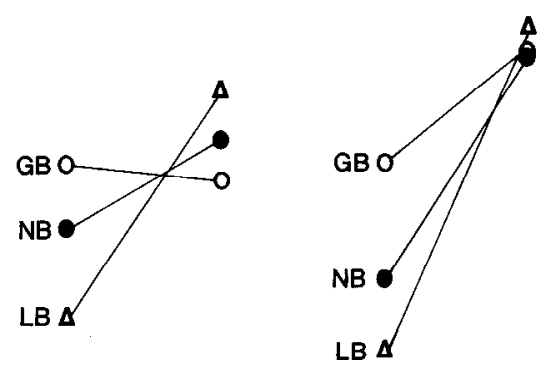

RTP $(\mathrm{N}=6)$

$\operatorname{RTP}_{2}(\mathrm{~N}=3)$

\section{Target Level}

Figure 3. Mean reaction time for the No-Bias $(N B)$, Global-bias $(G B)$, and Local-bias $(L B)$ conditions as a function of target level $(L o c a l$, Global) for the control group, left superior temporal gyrus group $(L S T G)$, left inferior parietal lobule group (LIPL), and right temporal parietal group $(R T P)$. The $\mathrm{RTP}_{2}$ group includes 3 RTP patients with large lesions encompassing both STG and IPL.

time to global and local levels per se, as indicated by the Target level $\times$ Group interaction. Controls and LIPL subjects showed no significant local or global reaction time advantage (although in both cases the means were in the local direction for the Nobias condition). In contrast, there was a significant global advantage for the LSTG group that was in the opposite direction from controls and resulted in a Target level $\times$ Group interaction when controls and LSTG were directly compared, $F(1,11)=$ $5.92, p<0.04$. As can be seen in Table 2, all 5 subjects in the LSTG group showed a global advantage in the baseline No-bias condition. LIPL and controls did not differ in this respect. Thus, the local deficits previously reported in left hemisphere patients as discussed in the introduction were observed only in the LSTG group and not in the LIPL group in the present study. This was further confirmed by the fact that the reaction time advantage for global or local targets was in the opposite direction for the LSTG and RTP groups, resulting in a Target level $\times$ Group interaction, $F(1,9)=5.58, p<0.05$. The LIPL and RTP groups did not differ in this respect.

Since the RTP group could not be divided into subgroups in the same way as the left hemisphere subjects, we could not evaluate analogous regions in these subjects. However, reaction time for the RTP group as a whole favored the local level, and differed from controls when the target was an $\mathrm{S}$ but, for unknown reasons, not when it was an $\mathrm{H}$ (see Fig. 4). Unlike the other comparisons with controls, there was a Target level $\times$ Target letter $\times$ Group interaction when controls and RTP were com-

\begin{tabular}{|c|c|c|c|c|c|c|}
\hline \multirow[b]{3}{*}{ Group } & \multicolumn{6}{|c|}{ Reaction time (msec) } \\
\hline & \multicolumn{2}{|c|}{ Global bias } & \multicolumn{2}{|c|}{ No bias } & \multicolumn{2}{|c|}{ Local bias } \\
\hline & Local & Global & Local & Global & Local & Global \\
\hline \multicolumn{7}{|l|}{ RTP } \\
\hline $\mathrm{KE}$ & 709 & 629 & 702 & 729 & 585 & 871 \\
\hline RR & 893 & 592 & 799 & 635 & 719 & 695 \\
\hline GB & 865 & 760 & 946 & 878 & 873 & 897 \\
\hline RM & 978 & 1281 & 837 & 1176 & 775 & 1062 \\
\hline $\mathrm{JC}$ & 833 & 682 & 593 & 670 & 474 & 706 \\
\hline HS & 611 & 858 & 634 & 973 & 550 & 1099 \\
\hline \multicolumn{7}{|l|}{ LIPL } \\
\hline RG & 470 & 498 & 552 & 488 & 495 & 541 \\
\hline ES & 614 & 712 & 553 & 627 & 546 & 729 \\
\hline FO & 575 & 521 & 551 & 540 & 540 & 533 \\
\hline FA & 715 & 615 & 562 & 683 & 534 & 631 \\
\hline MK & 704 & 605 & 552 & 581 & 540 & 643 \\
\hline \multicolumn{7}{|l|}{ LSTG } \\
\hline RW & 1589 & 827 & 1224 & 968 & 1225 & 1100 \\
\hline DL & 987 & 791 & 918 & 812 & 862 & 915 \\
\hline LG & 1043 & 851 & 928 & 787 & 802 & 1286 \\
\hline BB & 1261 & 947 & 1245 & 1051 & 855 & 962 \\
\hline RH & 1154 & 630 & 1188 & 687 & 851 & 717 \\
\hline
\end{tabular}




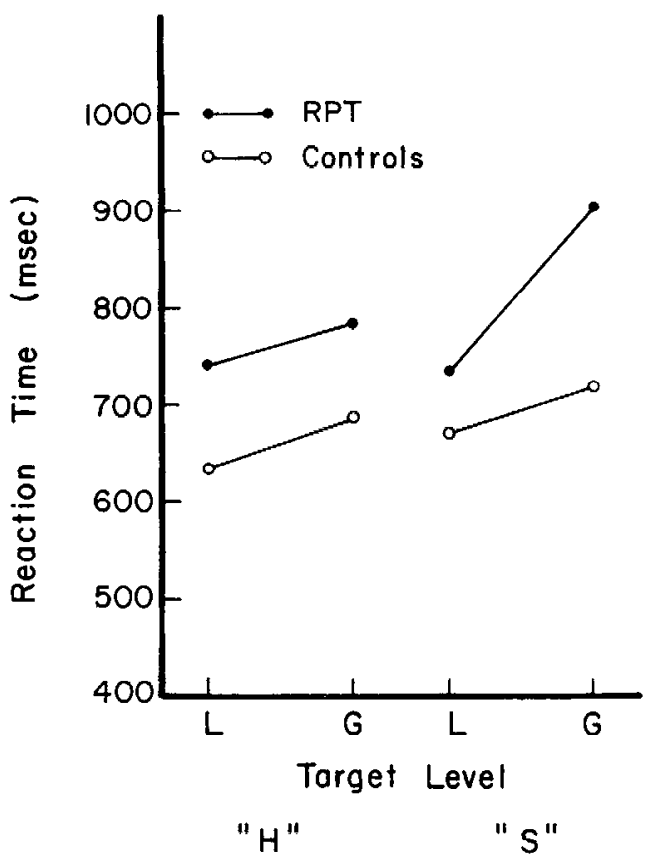

Figure 4. Mean reaction time to the target " $H$ " and " $S$ " as a function of target level ( $L$, local; $G$, global) for the right temporal parietal group $(R T P)$ and controls.

pared, $F(1,13)=5.59, p<0.035$. Three subjects in this group had small lesions, while 3 had large lesions. When we compared only the 3 RTP subjects with large lesions including both STG and IPL regions $\left(\mathrm{RTP}_{2}\right)$ to controls, there was, in fact, a significantly enhanced local advantage whether the target was an $\mathrm{H}$ or an S. Thus, the RTP as a whole showed a significantly enhanced local advantage compared with controls with only one target, while $\mathrm{RTP}_{2}$ subjects were different from controls independent of the target identity. Perhaps S's were simply more difficult to process than H's, and the anticipated change in RTP would be expected first with a more difficult stimulus, especially with smaller lesions. There was some weak support for this in the control group. There was a small advantage in reaction time when responding to $\mathrm{H}$ compared with $\mathrm{S}$, but this effect did not reach significant levels.

It should also be noted that reaction time to global targets did not vary as the target probabilities changed in $\mathrm{RTP}_{2}$ subjects, while reaction time to local targets did. Not much can be said of this trend since the Target level $\times$ Bias condition $\times$ Group interaction did not reach significant levels when $\mathrm{RTP}_{2}$ and controls were compared.

\section{Errors}

Errors were less than $6 \%$ for controls, and there was no indication of a speed-accuracy trade-off between errors and reaction times. A speed-accuracy trade-off would indicate that the effects could be attributed to a simple criterion or strategy shift. The correlation between reaction time and errors for controls was positive, $r=+0.50$. That is, more errors were made when reaction times were longer and fewer were made when reaction times were shorter. A speed-accuracy trade-off would result in a negative correlation between reaction time and errors. The error rate for the patient groups was $13 \%$ for LSTG, $14 \%$ for LIPL, and $8 \%$ for RTP. Again, no speed-accuracy trade-offs were observed within each group, $r=+0.70$ for LSTG, +0.10 for LIPL, and +0.47 for RTP. Recall that only the correct responses were included in the reaction time data. Errors were not analyzed further.

The nearly equal error rates for LIPL and LSTG patients indicates that the overall difference in reaction time between these groups could not be attributed to a speed-accuracy tradeoff. However, the faster responses by LIPL than controls could be due to this type of strategy shift because LIPL made nearly 3 times as many errors as controls. Because there was no speedaccuracy trade-off within LIPL, this could not explain the flattened pattern of response over the bias conditions, but it does make the differences between controls and LIPL in overall reaction time difficult to interpret. Another possible explanation for this difference is that some inhibitory mechanism was disrupted in LIPL, the nature of which is obscure at this point. ${ }^{3}$

\section{Discussion}

There were 3 main findings in the present study. First, controlled attentional processes were normal in patients with lesions centered in the LSTG but were disrupted in patients with lesions centered in the LIPL. Second, response time to both global and local targets was slower in LSTG patients than in controls or LIPL patients, suggesting visual discrimination difficulties. Third, the observed asymmetries in reaction time to global and local targets occurred between LSTG and RTP and not between LIPL and RTP, suggesting a perceptual locus of the global/local asymmetry. These results converge with several lines of investigation. First, they are consistent with studies employing monkeys that have revealed 2 different functional visual cortical pathways, one through the temporal lobe associated with visual discrimination and one through the parictal lobc associated with spatial relations and spatial attention. Second, they are consistent with findings from cognitive psychology that have implicated both attentional and perceptual mechanisms in analyzing global/local patterns. Third, they are consistent with studies in neuropsychology that have found global deficits in right hemispheredamaged patients and local deficits in left hemisphere-damaged patients. The implications for each of these areas will be discussed in turn.

\section{Relevance for studies of neuroscience}

The finding that attention was impaired in the LIPL group but not in the LSTG group is consistent with results from studies of the inferior parietal lobe in higher primates that have indicated an attentional role for this region (Lynch et al., 1977; Mountcastle, 1978; Wurtz et al., 1980; Bushnell et al., 1981). Relevant stimuli draw attention, and relevant stimuli enhance the response of cells in the inferior parietal lobe independent of eye movements. Posner et al.'s (1984) findings in humans also point to an attentional role for the parietal lobe, although the best predictor of attentional deficits in their study appeared to be lesions in the superior parietal lobe. All of these studies

\footnotetext{
$\overline{3}$ Reaction time differences between groups are potentially problematic because the changes in the magnitude of the Bias condition $\times$ Target level interactions could be due to an overall change in the rate of processing that would artificially increase or decrease the crossover effect. For this reason we performed a natural logarithmic transformation on the individual subject medians and reanalyzed the data using these values. This transformation did not change any of the major results. LSTG still differed from controls in the favored level, $F(1,12)=8.64, p$ $<0.01$, and this did not interact with biasing condition, $F<1$. Conversely, the 3-way interaction between Group; Target level, and Bias condition was significant when LIPL and controls were compared, $F(2,24)=4.41, p<0.03$, but the Target level $\times$ Group interaction was not, $F<1$. RTP and LSTG still differed in the favored level as would be expected, $F(1,9)=5.30, p<0.05$.
} 
examined the process that controls the focus of attention on a particular location rather than as a process that divides attention over extended areas of the visual field. Thus, the present results in combination with studies of selective attention in monkey and man suggest that the inferior parietal lobe is involved not only in attentional allocation to discrete locations in the visual field but also is critical in the division of attention over larger segments of the visual field. Specifically, under conditions in which normals and LSTG groups were able to control attention to larger global and smaller local levels by reallocating attentional resources, LIPL patients were markedly impaired.

There are alternative explanations for the lack of a bias condition effect in the LIPL group. First, these subjects may not have been sensitive to the changes in the probability of targets at the 2 levels between blocks. They knew there were 2 levels, and they may simply have divided their attention equally between the 2 levels unaware of the fact that a target was more likely to occur at one level or the other in different blocks of trials. Although this explanation cannot be discounted entirely, there is no evidence either clinically or experimentally that patients with lesions in this area should have difficulty detecting such probability distributions. In contrast, there is a great deal of evidence that they have difficulty with spatial attention.

The second alternative is that there is a floor effect present. This is not a likely interpretation since response time to the global level in the Global-bias condition had room to improve but did not. In addition, there was a large capacity to detect slowing in response times when a target occurred only $25 \%$ of the time at a given level. Although in both LB and GB there was a trend for some slowing in reaction time relative to the No-bias condition, it was not significant and was certainly not as large as in the control or LSTG groups.

We conclude, then, that the lack of a biasing condition effect in LIPL patients was due to a deficit in controlling the allocation of attentional resources. Although we cannot conclude that these patients were completely unable to control the allocation of attention to the 2 levels, since this would be based on a null effect with a small number of subjects, we can conclude that this process was significantly impaired relative to controls and to the LSTG group.

We also found hemisphere differences in relative reaction time to global and local levels. This effect was restricted to superior temporal regions in the left and was more pronounced with large lesions involving both STG and IPL in the right. Hemisphere differences have not been a major focus in studies of temporalparietal regions in monkey. Ablations are often made on the same side across subjects, and recordings from studies measuring single cells are often taken from one hemisphere or the other, so conclusions are typically made without considering hemisphere differences. It is therefore unknown to what extent hemisphere differences would occur in STG regions, but the present data do present the possibility that they may also be found in nonhuman primates.

As regions projecting to STG have been implicated in higherlevel visual processing, it is likely that the global/local asymmetry is directly perceptual in nature. In monkey there are strong projections from visual area MT in the superior temporal sulcus to surrounding STG regions, and these STG regions have receptive fields that are sensitive to visual stimuli (Desimone and Ungerleider, 1986; Ungerleider and Desimone, 1986). In addition, there is increasing evidence that posterior parietal and posterior temporal regions form a neural network for visuo- spatial analysis (Ungerleider and Mishkin, 1982; Seltzer and Pandya, 1984; Desimone and Ungerleider, 1986). Although the type of stimulus patterns used in physiological and ablation studies are typically much simpler than those used here, the contribution of temporal regions to visual processing is well supported. Thus, the neurophysiological evidence is consistent with our conclusion that the global/local dissociation observed in the present study is directly perceptual in nature.

In addition, the present results are consistent with the 2 visual pathway model of Ungerleider and Mishkin (1982) based on ablation studies in monkeys. Ungerleider and Mishkin proposed that the function of the inferior temporal pathway is visual discrimination, while the function of the inferior parietal pathway is spatial. In the present experiment, LSTG subjects had difficulty discriminating all targets (rcaction time was longcr for both global and local targets), although local targets were more affected than global ones. No deficit in distributing attention to the 2 levels was observed in this group. In contrast, LIPL subjects had no difficulty with discriminating the targets but did not allocate attention normally.

The present results are perhaps surprising considering that lesions between adjacent areas in humans cannot be isolated to the extent that they can by ablation studies in monkeys. In the present study there was substantial lesion overlap in the LSTG and LIPL groups. This situation was unavoidable and surely increased variability in performance. Despite the lesion overlap in the 2 groups, the data in the present study were systematic, reliable, and consistent with previous findings in humans and other animals. These data, in fact, demonstrate that it is quite possible to obtain meaningful differences in humans with lesions centered only a few centimeters apart by considering the area of overlapping damage. Certainly, lesion reconstruction employing saggital and axial MRI scans would enhance delineation of gyral topography and could reveal differences in the 2 groups that were not apparent by CT.

\section{Relevance for cognitive psychology}

The main reason that cognitive psychology has been interested in the processing of global/local patterns is that detection or response times favoring global targets suggest that perceptual organization begins with an overall analysis of a visual scene (the theory of global precedence originally advanced by the Gestalt psychologists) followed by a parsing of the scene into elements. Conversely, performance favoring local targets would suggest that perceptual organization begins with elements that are then combined to form an overall scene. There has been a long and extensive debate concerning which of these 2 alternatives most closely describes perceptual function, but to date there has been little resolution of the question (see Robertson, 1986, for an overview). The present results suggest that the cortical perceptual system processes global and local level information independently and in parallel. Since encoding targets at global and local levels can be separately affected by lesions in different areas of the human brain, global and local patterns do not have to compete for the resources of a single mechanism. In a world where milliseconds count, it would make a great deal of sense to have both global and local information available as early as possible, since either one could reflect danger or reward. The easiest way to accomplish this would be to have each level processed independently. The present data do not reveal how the 2 levels are integrated as they must be at some point in time (although see Lamb et al., 1988), but their asymmetrical rep- 
resentation in initial processing is well supported by the present findings.

A second issue in cognitive psychology has been the role of perception and attention in global and local analysis. There are really 2 types of perceptual explanations, one proposing that known retinal or sensory functions can account for global or local precedence, and one proposing a higher-order cognitive explanation. There are also attentional models that have generally suggested that the cognitive mechanism producing a global or local advantage is attentional. One issue, then, is whether sensory processes can account for all performance differences, and the other issue concerns whether central processes are perceptual or attentional (see Kubovy and Pomerantz, 1981 for several chapters concerning these issues). The present results demonstrate that global and local performance differences can be affected by lesions beyond primary visual cortex and clearly beyond the sensory receptor stage. They further demonstrate that the performance differences can be due to 2 different higherlevel mechanisms, one a controlled attentional process associated with inferior parietal regions and another more passive, automatic function associated with temporal regions.

\section{Relevance for neuropsychology studies}

The findings in the present study are consistent with those of Robertson and Delis (1986) and Delis et al. (1986), who reported that responses to local information were more disrupted in patients with lesions in the left hemisphere, and responses to global information were more disrupted with lesions in the right hemisphere. The findings also demonstrate that the asymmetry can be observed in early processing stages and suggest a perceptual locus. It is not known what stimulus parameters differentiate the encoding of global and local targets, although spatial frequencics have becn proposed as one possibility (Sergent, 1982; Shulman et al., 1986; Shulman and Wilson, 1987). There are, of course, several other parameters in global/local patterns that could be differentially encoded (e.g., size, density, number of elements, etc.), but these have not been sufficiently explored to make any meaningful statement about their role in the asymmetries observed here.

The results also support clinical observations that so-called "details" are disrupted by lesions in the left hemisphere, while "configurations" are disrupted by lesions in the right hemisphere (Kaplan, 1976). ${ }^{4}$ There are important qualifying statements that must be made in light of the present findings. Lesions centered in the left inferior parietal lobe did not produce disruptions in baseline local processing, while lesions centered in the left superior temporal gyrus did. Lesions in the RTP group, including both STG and IPL, produced disruptions in global processing. Furthermore, the interaction that revealed the asymmetry was found between LSTG and RTP and not between LIPL and RTP. It may have been the case that the procedures we used were not sensitive enough to measure subtle local deficits that may, in fact, have been present in LIPL patients. This does not seem likely because, if anything, there was a tendency toward a local advantage in these patients. It is more likely that many previous neuropsychological studies of this effect have not noticed the intrahemisphere effects due to their use of groups with large

\footnotetext{
${ }^{4}$ The words "detail" and "configuration" are highly ambiguous. In a stimulus with global and local levels, there are details (e.g., lines and curves) in the stimulus at both levels. Likewise, the local pattern is a configuration (e.g., a letter), as is the global pattern. For clinical purposes, these terms may be useful, but there has been no adequate operational definition of them in the scientific literature.
}

lesions encompassing several cortical areas, and, of course, visual field presentation in normals cannot differentiate intrahemispheric effects.

The present data are consistent with previous findings in neuropsychology in the sense that processing global and local information can be affected independently by lesions in the right and left hemispheres. More importantly, we have shown quite clearly that this asymmetry can be observed in the first few hundred milliseconds of analyzing a stimulus, depends on the specific intrahemispheric cortical area damaged, and persists for well over a year post injury. Furthermore, this effect is consistent with several lines of evidence pointing to a perceptual deficit associated with the multimodal superior temporal gyrus and could not be attributed to controlled attentional processes.

\section{References}

Bushnell, M. C., M. E. Goldberg, and D. L. Robinson (1981) Behavioral enhancement of visual responses in monkey cerebral cortex. I. Modulation in posterior parietal cortex related to selective visual attention. J. Neurophysiol. 46: 755-772.

DeArmond, S. J., M. M. Fusco, and M. M. Dewey (1976) Structure of the Human Brain, Oxford U. P., New York.

Delis, D. C., L. C. Robertson, and R. Efron (1986) Hemispheric specialization of memory for visual hierarchical stimuli. Neuropyschologia 24: 205-214.

Desimone, R., and L. G. Ungerleider (1986) Multiple visual areas in the caudal superior temporal sulcus of the Macaque. J. Comp. Neurol. 248: $164-189$

Frey, R. T., D. L. Woods, R. T. Knight, D. Scabini, and C. C. Clayworth (1987) Defining functional cortical areas with "averaged" CT scans. Paper presented at Society for Neuroscience, New Orleans.

Goldberg, M. E. (1982) Moving and attending in visual space: Single cell mechanisms in the monkey. In Spatial Abilities: Developmental and Physiological Foundations, M. Potegal, ed., pp. 277-300, Academic, New York.

Hoffman, J. (1980) Interaction between global and local levels of form. J. Exp. Psychol.: Human Percept. Perform. 6: 222-234.

Kahneman, D., and A. Treisman (1982) Changing views of attention and automaticity. In Varieties of Attention, R. Parasuraman and D. R. Davies, eds., pp. 29-61, Academic, New York.

Kaplan, E. (1976) The role of the noncompromised hemisphere in focal organic disease. Paper presented at the American Psychological Association meeting, Washington, D.C.

Keppel, G. (1973) Design and Analysis: A Researcher's Handbook, Prentice-Hall, Englewood Cliffs, NJ.

Kinchla, R. A., and J. Wolfe (1979) The order of visual processing: "top down," "bottom up," or "middle out." Percept. Psychophys. 25: 225-231.

Kinchla, R. A., V. Solis-Macias, and J. Hoffman (1983) Attending to different levels of structure in a visual image. Percept. Psychophys. 33: $1-10$.

Knight, R. T., D. Scabini, D. L. Woods, and C. C. Clayworth (1987a) Effects of lesions of superior temporal gyrus or inferior temporal lobe on temporal and vertex components of the human AEP. Soc. Neurosci. Abstr. 13: 331.

Knight, R. T., D. Scabini, D. L. Woods, and C. C. Clayworth (1987b) Differential effects of parietal and temporo-parietal lesions on human N200 \& P300. Soc. Neurosci. Abstr. 22: 521.

Kubovy, M., and J. R. Pomerantz, eds. (1981) Perceptual Organization, Erlbaum, Hillsdale, NJ.

Lamb, M. R., and L. C. Robertson (1987) Effect of acute alcohol on attention and the processing of hierarchical patterns. Alc. Clin. Exp. Res. 11: 243-248.

Lamb, M. R., and L. C. Robertson (1988) The processing of hierarchical stimuli: Effects of retinal locus, locational uncertainty and stimulus identity. Percept. Psychophys. 44: 172-181.

Lamb, M. R., L. C. Robertson, and R. T. Knight (1988) Effects of right and left temporal parietal lesions on the processing of global and local patterns in a selective attention task. Neuropsychologia (in press).

Lynch, J. C. (1980) The functional organization of posterior parietal association cortex. Behav. Brain Sci. 3: 485-534. 
Lynch, J. C., V. B. Mountcastle, W. H. Talbot, and T. C. T. Yin (1977) Directed visual attention. J. Neurophysiol. 40: 362-389.

Martin, M. (1979) Local and global processing: The role of sparsity. Memory Cog. 7: 476-484.

Mesulam, M. M. (1981) A cortical network for directed attention and unilateral neglect. Ann. Neurol. 10:309-325.

Mountcastle, V. B. (1978) Brain mechanisms for directed attention. J. R. Soc. Med. 71: 14-28.

Navon, D. (1977) Forest before trecs: The precedence of global features in visual perception. Cog. Psychol. 9: 353-383.

Newcombe, F., and W. R. Russell (1969) Dissociated visual perceptual and spatial deficits in focal lesions of the right hemisphere. J. Neurol. Neurosurg. Psychiatry 32: 73-81.

Palmer, S. E. (1982) Symmetry, transformation and the structure of perceptual systems. In Organization and Representation in Perception, J. Beck, ed., pp. 95-144, Erlbaum, Hillsdale, NJ.

Parasuraman, R., and D. R. Davies, eds. (1984) Varieties of Attention, Academic, New York.

Pohl, W. (1973) Dissociation of spatial discrimination deficits following frontal and parietal lesions in monkey. J. Comp. Physiol. Psychol. 82: 227-239.

Pomerantz, J. R. (1983) Global and local precedence: Selective attention in form and motion perception. J. Exp. Psychol.: General 112: 516-540.

Posner, M. I., and R. D. Rafal (1987) Cognitive theories of attention and the rehabilitation of attentional deficits. In Neuropsychological Rehabilitation, M. Meier, A. Benton, and L. Diller, eds., pp. 182201, Guilford, New York.

Posner, M. I., J. A. Walker, F. J. Friedrich, and R. D. Rafal (1984) Effects of parietal injury on covert orienting of attention. J. Neurosci. 4: 1863-1874.

Robertson, L. C. (1986) From gestalt to neo-gestalt. In Approaches to Cognition: Contrasts and Controversies, T. J. Knapp and L. C. Robertson, eds., pp. 159-188, Erlbaum, Hillsdale, NJ.
Robertson, L. C., and D. C. Delis (1986) "Part-whole" processing in unilateral brain damaged patients: Dysfunction of hierarchical organization. Neuropsychol. 24: 363-370.

Robertson, L.C ., and S. E. Palmer (1983) Holistic processes in the perception and transformation of disoriented figures. J. Exp. Psychol.: Human Percept. Perform. 9: 203-214.

Schneider, W., and R. M. Shiffrin (1977) Controlled and automatic human information processing: I. Detection, search and attention. Psychol. Rev. 84: 1-66.

Seltzer, B., and D. N. Pandya (1984) Further observations on parietotemporal connections in the rhesus monkey. Exp. Brain Res. 55: 301312 .

Sergent, J. (1982) The cerebral balance of power: Confrontation or cooperation? J. Exp. Psychol.: Human Percept. Perform. 8: 253-272.

Shiffrin, R. M., and W. Schneider (1977) Controlled and automatic human information processing: II. Perceptual learning, automatic attending and a general theory. Psychol. Rev. 84: 127-190.

Shulman, G. L., and J. Wilson (1987) Spatial frequency and selective attention to local and global information. Perception 16:89-101.

Shulman, G. L., M. A. Sullivan, K. Gish, and W. J. Sakoda (1986) The role of spatial-frequency channels in the perception of local and global structure. Perception 15: 259-273.

Ungerleider, L. G., and R. Desimone (1986) Cortical connections of visual area MT in the Macaque. J. Comp. Neurol. 248: 190-222.

Ungerleider, L. G., and M. Mishkin (1982) Two cortical visual systems. In Analysis of Visual Behavior, D. J. Ingle, M. A. Goodale, and R. J. W. Mansfield, eds., pp. 549-585, MIT Press, Cambridge, MA.

Wurtz, R. H., M. E. Goldberg, and D. L. Robinson (1980) Behavioral modulation of visual responses in the monkey: Stimulus selection for attention and movement. Prog. Psychobiol. Physiol. Psychol. 9: 4383.

Zeki, S. M. (1974) Functional organization of a visual area in the posterior bank of the superior temporal sulcus of the rhesus monkey. J. Physiol. (Lond.) 236: 549-573. 